\title{
Treatment Options for Class III Malocclusion in Growing Patients with Emphasis on Maxillary Protraction
}

\author{
Zeinab Azamian and Farinaz Shirban \\ Torabinejad Dental Research Center, Department of Orthodontics, School of Dentistry, \\ Isfahan University of Medical Sciences, Isfahan 81746-73461, Iran \\ Correspondence should be addressed to Farinaz Shirban; shirban123@yahoo.com
}

Received 29 December 2015; Revised 14 March 2016; Accepted 27 March 2016

Academic Editor: Kazuhisa Bessho

Copyright ( 2016 Z. Azamian and F. Shirban. This is an open access article distributed under the Creative Commons Attribution License, which permits unrestricted use, distribution, and reproduction in any medium, provided the original work is properly cited.

\begin{abstract}
It is very difficult to diagnose and treat Class III malocclusion. This type of malocclusion involves a number of cranial base and maxillary and mandibular skeletal and dental compensation components. In Class III malocclusion originating from mandibular prognathism, orthodontic treatment in growing patients is not a good choice and in most cases orthognathic surgery is recommended after the end of growth. Approximately 30-40\% of Class III patients exhibit some degree of maxillary deficiency; therefore, devices can be used for maxillary protraction for orthodontic treatment in early mixed dentition. In cases in which dental components are primarily responsible for Class III malocclusion, early therapeutic intervention is recommended. An electronic search was conducted using the Medline database (Entrez PubMed), the Cochrane Collaboration Oral Health Group Database of Clinical Trials, Science Direct, and Scopus. In this review article, we described the treatment options for Class III malocclusion in growing patient with an emphasis on maxillary protraction. It seems that the most important factor for treatment of Class III malocclusion in growing patient is case selection.
\end{abstract}

\section{Introduction}

Etiologic factors for Class III malocclusions include a wide spectrum of skeletal and dental compensation components [1]. The condition might be characterized by mandibular prognathism, maxillary retrognathism, retrusive mandibular dentition, protrusive maxillary dentition, and a combination of the above [2].

Clinically, Class III malocclusion is in two forms: (a) "pseudo or functional Class III," due to an early interference with the muscular reflex of mandibular closure and (b) the "true Class III" [3].

The etiology of Class III malocclusion is multifactorial, with genetic, ethnic, environmental, and habitual components [4]. It was believed until 1970 that only the mandible is responsible for Class III malocclusion [5]; however, almost $30-40 \%$ of patients exhibit some degree of maxillary deficiency [6].

Different ethnic groups exhibit different prevalence rates of Class III, with different methods of classification being used. The prevalence rate was reported to be around $1-3 \%$ in the Caucasians and around $13-14 \%$ among the Chinese and Japanese [7-11]. In the Asian population the majority of patients exhibit midface deficiency [12]. It has been reported that more than $60 \%$ of Class III malocclusion cases are due to skeletal discrepancies [13].

Final and definitive diagnosis of skeletal Class III malocclusion is based on the following:

(a) Verification of the normal centric position with the habitual position.

(b) Presence or absence of a familial predisposition.

(c) Cephalometric parameters, including a decrease in SNA, negative ANB, mandibular protrusion, obtuse gonial angle, and great LAFH.

(d) Incisor relationship [14].

This study was undertaken to evaluate different types of devices used to correct Class III malocclusion in growing 
TABLE 1: Appliances for correction of class III malocclusion in growing patients.

\begin{tabular}{|c|c|c|c|}
\hline \multirow{7}{*}{ Intraoral appliances } & Fixed & Class III elastic with skeletal anchorage [1] & Skeletal effect \\
\hline & \multirow{6}{*}{ Removable } & Modified Balters’ Bionator III [15] & Dental effect \\
\hline & & Frankel III [17] & Skeletal/dental effect \\
\hline & & Reverse twin block [40] & Dental effect \\
\hline & & Eschler/progenic appliance (removable mandible retractor) [18] & Dental effect \\
\hline & & Double-piece corrector [19] & Dental effect \\
\hline & & Tandem appliance [20] & Skeletal/dental \\
\hline \multirow{3}{*}{ Extraoral appliances } & & Chin cap [21] & Skeletal \\
\hline & & Face mask $[24,25]$ & Skeletal \\
\hline & & Headgear for mandibular arch $[22,23]$ & Skeletal/dental \\
\hline
\end{tabular}

patients with an emphasis on devices used for maxillary protraction.

\section{Search Strategy}

A computerized search was carried out using the Medline database (Entrez PubMed, http://www.ncbi.nlm.nih.gov/), the Cochrane Collaboration Oral Health Group Database of Clinical Trials (http://www.cochrane.org/), Science Direct (http://www.sciencedirect.com/), and Scopus (http://www .scopus.com/) from 1956 to 2015 and from 20th February to 4th April 2015. The terms used included maxillary deficiency, Class III malocclusion, maxillary protraction, and bone anchors. During the preliminary search, 250 articles were selected based on the article titles and then, based on the aims of this study, 96 articles were evaluated.

\section{Appliances}

The options for correction of Class III malocclusion in growing patients consist of two principal categories: intraoral appliances and extraoral appliances (Table 1).

\subsection{Intraoral Appliances}

3.1.1. Class III Elastics with Skeletal Anchorage. Four miniplates are inserted in the left and right infrazygomatic crest of the maxillary buttress and between the lower left and right lateral incisors and canines (Figure 1). A mucoperiosteal flap is elevated and the miniplates are placed in the underlying bone by miniscrews. The extension of the plates perforates the attached gingiva and they are loaded three weeks later with Class III elastics [1].

3.1.2. Bionator III. The reverse Bionator or Bionator III, a modified form of the traditional Bionator, is used in the treatment of Class III malocclusion cases. The modified Balters' Bionator III [15] exhibits differences from the original version, with deeper and wider lingual wings, acrylic vestibular lateral shields extending deep into the upper fornix, upper labial buttons, and upper incisor inclined plane. The construction bite is normally taken by gentle repositioning of the mandible in the centric relation. Patients are expected to wear such an appliance for a minimum of 22 hours a day [16].

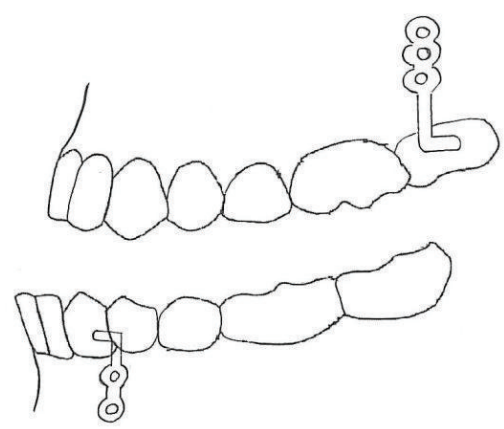

FIGURE 1: Class III elastic with skeletal anchorage.

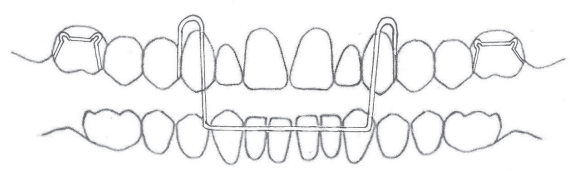

FIGURE 2: Eschler appliance.

3.1.3. Frankel III Functional Appliance. Frankel III functional appliance is made while the mandible is positioned posteriorly. It has pads to stretch the upper lip and periosteum forward that stimulates forward growth of maxilla [17].

3.1.4. Eschler Appliance. The Eschler appliance consists of 3 parts. The first part is a retention component such as Adams clasps for molars and intermolar auxiliary clasps for deciduous teeth and premolars. The second part is an Eschler labial bow made of a $0.9 \mathrm{~mm}$ wire (Figure 2). The third part is occlusal bite raising, made of acrylic resin measuring $2-3 \mathrm{~mm}$ in thickness. An expansion screw or spring can be added for some specific purposes [18].

3.1.5. Double-Plate Appliance. The double-plate appliance is an intraoral appliance containing angulated acrylic blocks, with an acrylic segment that contacts the lingual surfaces of lower incisors in order to prevent their retraction (Figure 3). This appliance is used with a face mask [19]. 


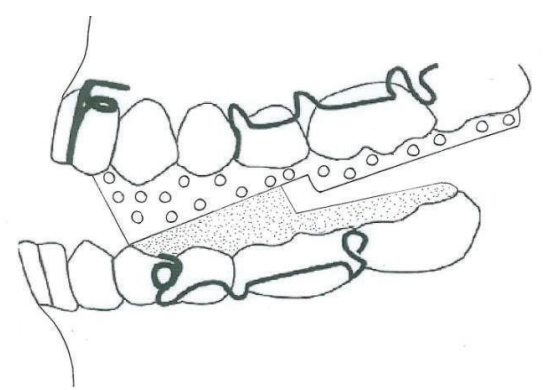

FIgURE 3: Double-plate appliance.

3.1.6. Tandem Appliance. The Tandem appliances are made up of three components. The upper appliance is fixed, with bands on deciduous second molars, a transpalatal arch, and palatal expansion arms and buccal arms for elastic traction. The lower appliance has bands on deciduous second molars, a lingual holding arch, a fixed bite plane for posterior occlusal coverage, and buccal face bow tubes (Figure 4). The outer bow of the headgear face bow has been modified to engage elastics and is inserted into the lower tubes [20].

\subsection{Extraoral Appliances}

3.2.1. Chin Cap. Chin cap is a useful appliance in growing patients that exhibit mandibular prognathism and short lower facial height. It has been shown that chin cap redirects mandibular growth, rotates the mandible backward, retards mandibular growth, and remodels the mandible [21]. It also increases the anterior facial height. It is particularly more useful for Asian children compared to Caucasians, which is attributed to their shorter facial height and greater protrusion of lower incisors, rather than to differences in their response to treatment [17].

3.2.2. Headgear for Mandibular Arch. Baccetti et al. [22] and Rey et al. [23] used the mandibular cervical headgear in growing Class III patients exhibiting mandibular prognathism. This treatment option results in distalization of mandibular molars and redirection of mandibular growth.

3.2.3. Face Mask. Orthopedic protraction of maxilla in Class III patients exhibiting maxillary retrusion and meso- or brachyfacial patterns proved effective [24, 25]. The most effective appliance in such cases is a face mask. However, there are some limitations in the use of a face mask, including patient compliance problems, dentoalveolar effect, limited protraction of maxilla ( $2-3 \mathrm{~mm}$ in $9-12$ months), and the possibility of relapse as a result of mandibular growth [2630].

Face masks have various clinical applications. The clinician may choose a Petit face mask or a Delaire type as an extraoral part of the appliance, opt for skeletal anchorage versus dental anchorage, or choose advancement with expansion in contrast to advancement without expansion. Here, we are going to review use of face masks in dental clinics.
Delaire face mask is commonly used for protraction of maxilla. The chin and forehead are used for extraoral anchorage [31]. This appliance might interfere with sleep or wearing eyeglasses [17]. Petit modified the Delaire face mask in 1983, incorporating a forehead and a chin pad that were connected with a heavy steel rod [32].

3.2.4. Protraction of Maxilla with Expansion and without Expansion. Use of rapid maxillary expansion (RME) has been recommended for protraction of maxilla. Some authors believe that expansion will disarticulate maxilla and initiate cellular response [33-35]. The appliance in the maxillary arch is a bonded or banded maxillary expander. The patient activates the expander once or twice a day until the desired transverse relationship is achieved [1]. Another protocol is the use of alternate rapid maxillary expansions and constrictions (Alt-RAMEC). Activation of expansion/constriction is $0.5 \mathrm{~mm}$ daily [36] to disarticulate the suture without overexpansion $[37,38]$. However, a meta-analysis found that protraction was the same with or without expansion [29].

3.2.5. Face Mask with Dental Anchorage. A routine protocol for face mask therapy is application of force to a removable appliance in the maxilla. There is consensus over application of force at $30^{\circ}$ angulation to the occlusal plane for minimum unwanted rotation of the maxilla. Forces of 300-600 g on each side are favorable. The skeletal results obtained with different amounts of force (300-500 g) are similar, resulting in $3^{\circ}$ increase in SNA [39].

3.2.6. Protraction Face Mask and Reverse Twin Block. Early treatment of Class III malocclusions with protraction face mask and reverse twin block (PFM and RTB) might be effective. The remaining growth will influence the long-term stability of these treatments [40].

\subsubsection{Face Mask with Skeletal Anchorage: Bone Anchor Maxillary Protraction (BAMP)}

(1) Face Mask with a Titanium Screw. Titanium screws have been successfully used as skeletal anchorage [41]. These screws do not require latency time for osseointegration, and treatment can be instituted immediately after insertion. In a case report, a lag titanium screw was applied as skeletal anchorage for protraction of maxilla. $800 \mathrm{~g}$ force per side was applied at a $30^{\circ}$ angle relative to the occlusal plane. The anterior nasal spine was advanced approximately $3 \mathrm{~mm}$ anteriorly, with stable improvement after a year [42].

(2) Face Mask with Onplant. In 1995 Block and Hoffman applied onplant as an anchorage for orthodontic purposes in animals [43]. The onplants were reported to tolerate forces up to $300 \mathrm{~g}$. In a different study onplants were used for application of force to the maxilla. Subsequent to a surgical operation for insertion of onplants $(7.7 \mathrm{~mm}$ hexagonal onplants) near the molar area (Figure 5), a vacuum-formed stent was used for 10 days. Osseointegration occurred over a period of 3-4 months. Then a $400 \mathrm{~g}$ force per side was 

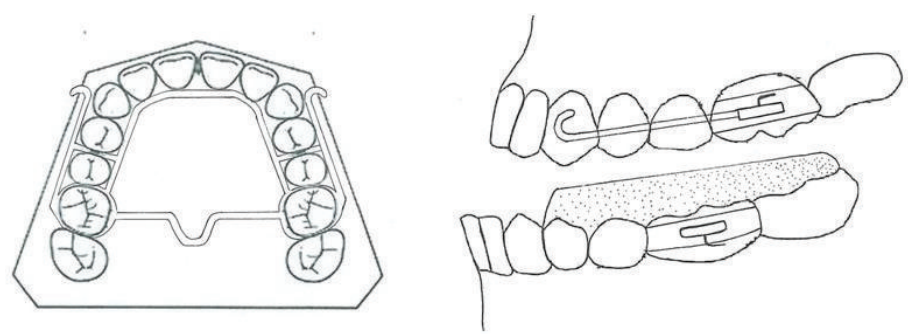

Figure 4: Tandem appliance.

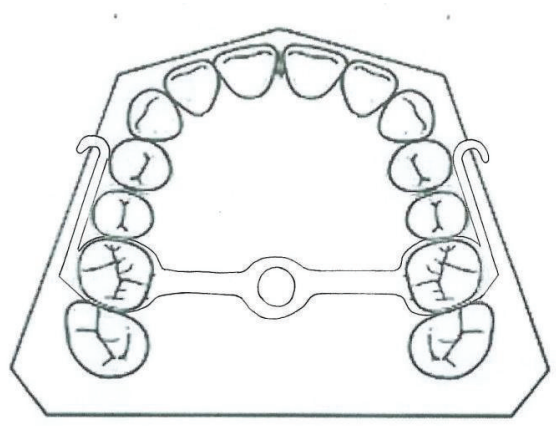

FIGURE 5: Face mask with skeletal anchorage.

transmitted to the hooks in the premolar area in the maxillary fixed appliance. The onplants, as a reference point, moved $2.9 \mathrm{~mm}$ horizontally and $2.9 \mathrm{~mm}$ vertically over a 12 -month period [44].

(3) Face Mask with Osseointegrated Implants. The first clinical use of titanium implants as an anchorage for maxillary protraction occurred in an animal study. These Brånemark implants withstood $600 \mathrm{~g}$ force per side and an $8 \mathrm{~mm}$ advancement of the maxilla was achieved [45]. In a different study, implants were used in the zygomatic process of the maxilla and a $400 \mathrm{~g}$ force per side was applied, resulting in a $4 \mathrm{~mm}$ advancement of the maxilla [46].

(4) Face Mask to an Ankylosed Primary Canine. Use of an intentionally ankylosed tooth is a proper technique for direct transmission of force for protraction of the maxilla. However, such teeth undergo resorption as their permanent successors erupt, restricting use of ankylosed teeth to young patients [47-49].

3.2.8. Corticotomy-Assisted Maxillary Protraction. Low-angle Class III patients who exhibit severe retrognathism of the maxilla, patients who have lost chance of orthopedic correction, and patients who refuse to undergo orthognathic surgeries are candidates for corticotomy-assisted maxillary protraction [50]. Sutural distraction osteogenesis versus osteotomy distraction osteogenesis for protraction of midface has already been used. Lefort III fractures have been used in the zygomaticofrontal suture. Distraction has been carried out with the use of heavy elastics [51].
Rachmiel et al. in 1999 [52] and Samchukov in 2001 [53] reported patients treated by an incomplete Lefort I osteotomy followed by face mask protraction. They reported 5-9 $\mathrm{mm}$ of maxillary protraction. In such a treatment modality a face mask is used for 5-7 days after surgery and a 1700-2000 g force is applied. Significant relapse of maxillary advancement was detected in a 6-year follow-up. However, well-preserved dental relationship was reported [54].

\section{Discussion}

Orthopedic treatments might prove effective in children with Class III malocclusion in the short term [55]. Several appliances are used for early treatment of skeletal Class III, including Bionator [15], Frankel (FR-III) [17], chin cup [21], double-plate appliance [19], Eschler appliance "progenic appliance" [18], and protraction face mask. Orthopedic protraction of the maxilla is a popular treatment modality, with some limitations, including problems with patient compliance, limited protraction of the maxilla $(2-3 \mathrm{~mm}$ in $9-12$ months), unwanted dentoalveolar effects, and the possibility of relapse as a result of late mandibular growth $[28,29,56]$.

Face mask therapy is effective in Class III, maxillarydeficient, deep-bite patients, and all the treated patients exhibit positive overjet after treatment. In a study, after face mask therapy, the maxilla continued to grow in the anterior direction in an amount equal to untreated Class III patients but less than that in untreated Class I patients; mandibular growth was similar in all the groups [57].

4.1. Age of Intervention (Face Mask Therapy). An important factor determining the success of treatment for Class III patients is treatment timing. It has been recommended that face mask therapy should be initiated at 6-8 years of age after eruption of maxillary permanent first molar and incisors, that is, early mixed dentition $[20,39,58,59]$. However, maxillary protraction with bone anchors and Class III elastics has been reported to be successful in the late mixed or permanent dentition phases [1].

4.2. Maxillary Protraction with or without Maxillary Expansion? In addition, rapid maxillary expansion (RME) has been recommended as a routine component of treatment for correction of Class III malocclusion, even in the absence of maxillary constriction because it disarticulates the maxilla and gives rise to cellular responses in the circummaxillary 
sutures, bringing about a more positive reaction to protraction forces $[34,35,60]$. Nevertheless, when used to enhance anterior movement of the maxilla during face mask therapy, preliminary RME does not appear to exert any effect on the efficacy of orthopedic treatment [61]. There are reports that use of RME alone might not properly disarticulate circummaxillary sutures and it might be better dealt with by Alt-RAMEC $[36,62,63]$. A meta-analysis showed similar results for protraction with or without expansion [29].

\subsection{Treatment Outcome for Face Mask Therapy with Dental} or Skeletal Anchorage. Cephalometric analyses have shown skeletal and dentoalveolar changes in face mask therapy. Skeletal changes include maxillary protrusive movement (SNA, N-perpA) and downward and backward rotation of the mandible (SNGoGN, SNGn, and LAFH), with a decrease in prognathism severity (SNB). Such changes induce favorable changes in the facial profile. Dentoalveolar changes mainly consist of linguoversion of mandibular incisors and labial inclination of maxillary incisors [64]. The forces used for maxillary protraction are usually applied to maxillary teeth. Therefore, there might be a significant mesial migration of maxillary teeth, possibly resulting in severe anterior crowding and in a decrease in the orthopedic effects of treatment, which might cause problems $[65,66]$. It has been demonstrated that the mandibular plane angle increases significantly during protraction face mask therapy [67-69]. Some of the complications associated with dental anchorage are resolved by skeletal anchorage. Recently, osseointegrated implants, titanium screws, onplants, and miniplates have been used as stable anchorage for maxillary protraction $[41,46,56,70]$.

The greatest disadvantage of palatal osseointegrated implants is that they can only be placed on the palate and are indicated for moving maxillary teeth. A palatal implant costs much higher than a miniscrew and miniplate and requires an osseointegration period [71].

Temporary anchorage devices for maxillary protraction have become very popular in recent years $[1,42,44,46,72]$.

A study showed an improvement in molar relation, which was not influenced by dental inclination and there was no significant amount of lingual inclination of the lower incisors in the BAMP group [1].

The proclination of mandibular incisors might be explained by the new posture of the tongue that acts on these incisors after correction of anterior crossbite [56, 73].

In Class III cases that are treated with skeletal anchorage, the amount of maxillary protraction might vary from 3.0 to $5.6 \mathrm{~mm}[36,73-75]$. The BAMP protocol can cause significantly larger maxillary advancement compared to the RME/FM therapy. BAMP results in $2.3-3 \mathrm{~mm}$ more maxillary protraction compared to face mask or rapid maxillary expansion [1]. BAMP protocol results in fewer vertical changes. Furthermore, these patients do not exhibit clockwise rotation of the mandible or dental compensation $[1,76]$. A $3.82 \mathrm{~mm}$ forward movement of the nose tip was reported as a result of the BAMP protocol [77]. The upper lip and lip sulcus also moved forward, and the soft tissue B point and pogonion moved backward during the protraction period, indicating improvements in the soft tissue profile in line with the underlying skeletal components during the protraction procedure $[56,78,79]$.

It has been reported that it is difficult to control the vertical growth and lower anterior facial height increases to some extent during maxillary protraction, with both dental and bone anchorage $[1,26,34,80]$. The low mandibular plane angle group exhibited a greater maxillary forward displacement and a larger increase in the maxillary body compared to the high mandibular plane angle group [81]. The craniomaxillary complex in the dental anchorage model is displaced forward along with rotation, and the amount of this rotation decreases gradually with an increase in the angle between the force vector and occlusal plane from 0 to 30 degrees. However, the craniomaxillary complex in the bone anchorage model is displaced forward along with rotation, and the rotation degree decreases gradually with an increase in the angle from 0 to 20 [82].

A cephalometric analysis of pharyngeal airway showed no significant changes in the oro- and nasopharyngeal sagittal airway dimensions in the face mask + bite block therapy group compared to the untreated Class III subjects [83].

Ghiz et al. [84] reported that four variables were significant in predicting successful treatment outcomes: (1) the position of the condyle relative to the cranial base; (2) ramus length; (3) mandibular length; and (4) gonial angle [85]. Three pretreatment cephalometric variables exhibited the highest predictive power in terms of discriminating gonial angle, nasion-A-pogonion angle, and ramus planeto-sella-nasion angle. Patients exhibiting larger values for these three measurements before treatment were categorized as the unstable group at the end of the observation period. Orthopedic treatment of Class III malocclusion might give rise to more favorable craniofacial adaptations when a patient's pretreatment cephalometric analyses reveal a short mandibular ramus (i.e., decreased posterior facial height) and a low mandibular plane angle [30].

4.4. Skeletal Analysis of Treatment Outcomes. An approach is to make use of a 3D skeletal color map of the superimposition on the anterior cranial base. The superimposition and semitransparent overlays show that bone-anchored maxillary protraction growth and treatment response lead to bone apposition at the anterior eminence of the TMJ, which correlates well with the posterior displacement of the anterior surface of the condyle, and the bone resorption of the posterior wall of the articular eminence correlates well with the posterior displacement of the posterior surface of the condyle. Mandibular shape, rather than the mandibular size, is under the influence of continuous intermaxillary traction [14]. Forward movement of the maxilla might be registered at the posterior nasal spine and at pterygomaxillary fissure points [72].

Another method is to use the patient's lateral cephalograms, which can be extracted from CBCT, in order to carry out TPS analysis [86]. TPS analysis deforms one landmark configuration into another, indicating that this change in shape is the deformation of a grid, at the same time making statistical comparisons possible. TPS has specific cephalometric indications to demonstrate differences in shape as a 
result of orthodontic treatment techniques or growth-related changes. In fact, TPS analysis has been used to study growth changes in treated and untreated subjects with different types of malocclusion $[87,88]$.

4.5. Retention and Follow-Up. In previous studies retention has been recommended after overjet and overbite correction from three months to two years during the night $[24,89$, 90]. Long-term follow-ups of maxillary protraction indicate a $25-33 \%$ chance of relapse to negative overjet after the completion of mandibular growth [30, 67, 91, 92]. It was concluded that relapse to a Class III pattern primarily results from mandibular growth rather than a relapse in the maxilla $[50,66]$. In another study, patients with larger values for the inclination of the mandibular ramus to the mandibular body (gonial angle) before treatment exhibited a higher probability of relapse at the end of the observation period $[30,93,94]$.

\section{Conclusion}

An important factor for treatment of Class III malocclusion in growing patient is the origin of malocclusion. The skeletal or dental origin of the malocclusion and in skeletal Class III malocclusions mandibular prognathism or maxillary deficiency are important for choosing early intervention and selection of the appliance for treatment. All appliances described in this paper can be useful when the clinicians use them in correct manner.

\section{Competing Interests}

The authors declare that there are no competing interests regarding the publication of this paper.

\section{References}

[1] L. Cevidanes, T. Baccetti, L. Franchi, J. A. McNamara Jr., and H. De Clerck, "Comparison of two protocols for maxillary protraction: bone anchors versus face mask with rapid maxillary expansion," The Angle Orthodontist, vol. 80, no. 5, pp. 799-806, 2010.

[2] M. Celikoglu and H. Oktay, "Effects of maxillary protraction for early correction of class III malocclusion," European Journal of Orthodontics, vol. 36, no. 1, pp. 86-92, 2014.

[3] R. E. Moyers, Handbook of Orthodontics, Year Book Medical Publishers, 1988.

[4] M. B. Khan and A. Karra, "Early treatment of class III malocclusion: a boon or a burden?” International Journal of Clinical Pediatric Dentistry, vol. 7, pp. 130-136, 2014.

[5] J. Mermigos, C. A. Full, and G. Andreasen, "Protraction of the maxillofacial complex," American Journal of Orthodontics and Dentofacial Orthopedics, vol. 98, no. 1, pp. 47-55, 1990.

[6] A. Arman, T. U. Toygar, and E. Abuhijleh, "Profile changes associated with different orthopedic treatment approaches in class III malocclusions," Angle Orthodontist, vol. 74, no. 6, pp. 733-740, 2004.

[7] M. Fu, D. Zhang, B. Wang, Y. Deng, F. Wang, and X. Ye, "The prevalence of malocclusion in China-an investigation of 25,392 children," Chinese Journal of Stomatology, vol. 37, no. 5, pp. 371-373, 2002.
[8] M. Irie and S. Nakamura, "Orthopedic approach to severe skeletal Class III malocclusion," American Journal of Orthodontics, vol. 67, no. 4, pp. 377-392, 1975.

[9] M. T. Bukhary, "Comparative cephalometric study of Class III malocclusion in Saudi and Japanese adult females," Journal of Oral Science, vol. 47, no. 2, pp. 83-90, 2005.

[10] M. Mouakeh, "Cephalometric evaluation of craniofacial pattern of Syrian children with Class III malocclusion," American Journal of Orthodontics and Dentofacial Orthopedics, vol. 119, no. 6, pp. 640-649, 2001.

[11] M. Celikoglu, S. Akpinar, and I. Yavuz, "The pattern of malocclusion in a sample of orthodontic patients from Turkey," Medicina Oral, Patologia Oral y Cirugia Bucal, vol. 15, no. 5, pp. e791-e796, 2010.

[12] G. V. Newman, "Prevalence of malocclusion in children six to fourteen years of age and treatment in preventable cases," Journal of the American Dental Association, vol. 52, no. 5, pp. 566-575, 1956.

[13] C. B. Staudt and S. Kiliaridis, "Different skeletal types underlying Class III malocclusion in a random population," American Journal of Orthodontics and Dentofacial Orthopedics, vol. 136, no. 5, pp. 715-721, 2009.

[14] H. De Clerck, T. Nguyen, L. K. De Paula, and L. Cevidanes, "Three-dimensional assessment of mandibular and glenoid fossa changes after bone-anchored Class III intermaxillary traction," American Journal of Orthodontics and Dentofacial Orthopedics, vol. 142, no. 1, pp. 25-31, 2012.

[15] A. Levrini and L. Levrini, "Il Bionator concetti classici e nuove acquisizioni," Rivista Italiana di Stomatologia, vol. 10, pp. 499506, 1993.

[16] G. Garattini, L. Levrini, P. Crozzoli, and A. Levrini, "Skeletal and dental modifications produced by the Bionator III appliance," American Journal of Orthodontics and Dentofacial Orthopedics, vol. 114, no. 1, pp. 40-44, 1998.

[17] W. R. Proffit, H. W. Fields Jr., and D. M. Sarver, Contemporary Orthodontics, Elsevier Health Sciences, Philadelphia, Pa, USA, 2014.

[18] M. R. de Almeida, R. R. de Almeida, P. V. P. Oltramari-Navarro, A. C. D. C. F. Conti, R. D. L. Navarro, and J. G. D. D. Camacho, "Early treatment of Class III malocclusion: 10-year clinical follow-up," Journal of Applied Oral Science, vol. 19, no. 4, pp. 431439, 2011.

[19] D. Gencer, E. Kaygisiz, S. Yüksel, and T. Tortop, “Comparison of double-plate appliance/facemask combination and facemask therapy in treating Class III malocclusions," Angle Orthodontist, vol. 85, no. 2, pp. 278-283, 2015.

[20] R. Sukh, G. P. Singh, and P. Tandon, "A new modified tandem appliance for management of developing Class III malocclusion," Contemporary Clinical Dentistry, vol. 4, no. 4, pp. 515-519, 2013.

[21] J. Sugawara, T. Asano, N. Endo, and H. Mitani, "Long-term effects of chincap therapy on skeletal profile in mandibular prognathism," American Journal of Orthodontics and Dentofacial Orthopedics, vol. 98, no. 2, pp. 127-133, 1990.

[22] T. Baccetti, D. Rey, D. Angel, G. Oberti, and J. A. McNamara Jr., "Mandibular cervical headgear vs rapid maxillary expander and facemask for orthopedic treatment of class III malocclusion," The Angle Orthodontist, vol. 77, no. 4, pp. 619-624, 2007.

[23] D. Rey, J. F. Aristizabal, G. Oberti, and D. Angel, "Mandibular cervical headgear in orthopedic and orthodontic treatment of Class III cases," World Journal of Orthodontics, vol. 7, no. 2, pp. 165-176, 2006. 
[24] A. L. Ramos, "Class III treatment using facial mask: stability after 10 years," Dental Press Journal of Orthodontics, vol. 19, no. 5, pp. 123-135, 2014.

[25] R. S. Tindlund and P. Rygh, "Maxillary protraction: different effects on facial morphology in unilateral and bilateral cleft lip and palate patients," Cleft Palate-Craniofacial Journal, vol. 30, no. 2, pp. 208-221, 1993.

[26] T. Baccetti, J. S. McGill, L. Franchi, J. A. McNamara Jr., and I. Tollaro, "Skeletal effects of early treatment of Class III malocclusion with maxillary expansion and face-mask therapy," American Journal of Orthodontics and Dentofacial Orthopedics, vol. 113, no. 3, pp. 333-343, 1998.

[27] P. K. Turley, "Managing the developing class III malocclusion with palatal expansion and facemask therapy", American Journal of Orthodontics and Dentofacial Orthopedics, vol. 122, no. 4, pp. 349-352, 2002.

[28] K.-S. Cha, "Skeletal changes of maxillary protraction in patients exhibiting skeletal class III malocclusion: a comparison of three skeletal maturation groups," The Angle Orthodontist, vol. 73, no. 1, pp. 26-35, 2003.

[29] J.-H. Kim, M. A. Viana, T. M. Graber, F. F. Omerza, and E. A. BeGole, "The effectiveness of protraction face mask therapy: a meta-analysis," American Journal of Orthodontics and Dentofacial Orthopedics, vol. 115, no. 6, pp. 675-685, 1999.

[30] T. Baccetti, L. Franchi, and J. A. McNamara Jr., "Cephalometric variables predicting the long-term success or failure of combined rapid maxillary expansion and facial mask therapy," American Journal of Orthodontics and Dentofacial Orthopedics, vol. 126, no. 1, pp. 16-22, 2004.

[31] J. D. Elai, "La croissance maxillaire: deductions therapeutiques," 1971.

[32] H. Petit, "Adaptation following accelerated facial mask therapy," in Clinical Alteration of the Growing Face, J. A. McNamara Jr., K. A. Ribbens, and R. P. Howe, Eds., Monograph no. 14, Craniofacial Growth Series, pp. 253-289, Center for Human Growth and Development, University of Michigan, Ann Arbor, Mich, USA, 1983.

[33] P. K. Turley, "Orthopedic correction of Class III malocclusion with palatal expansion and custom protraction headgear," Journal of Clinical Orthodontics, vol. 22, no. 5, pp. 314-325, 1988.

[34] Z. Altug and A. D. Arslan, "Skeletal and dental effects of a mini maxillary protraction appliance," The Angle Orthodontist, vol. 76, no. 3, pp. 360-368, 2006.

[35] B. H. Canturk and M. Celikoglu, "Comparison of the effects of face mask treatment started simultaneously and after the completion of the alternate rapid maxillary expansion and constriction procedure," Angle Orthodontist, vol. 85, no. 2, pp. 284-291, 2015.

[36] D. Kaya, I. Kocadereli, B. Kan, and F. Tasar, "Effects of facemask treatment anchored with miniplates after alternate rapid maxillary expansions and constrictions; a pilot study," Angle Orthodontist, vol. 81, no. 4, pp. 639-646, 2011.

[37] E. J.-W. Liou, "Effective maxillary orthopedic protraction for growing Class III patients: a clinical application simulates distraction osteogenesis," Progress in Orthodontics, vol. 6, no. 2, pp. 154-171, 2005.

[38] E. J.-W. Liou and W.-C. Tsai, "A new protocol for maxillary protraction in cleft patients: repetitive weekly protocol of alternate rapid maxillary expansions and constrictions," Cleft Palate-Craniofacial Journal, vol. 42, no. 2, pp. 121-127, 2005.

[39] E. Yepes, P. Quintero, Z. V. Rueda, and A. Pedroza, "Optimal force for maxillary protraction facemask therapy in the early treatment of class III malocclusion," European Journal of Orthodontics, vol. 36, no. 5, pp. 586-594, 2014.

[40] J. Seehra, P. S. Fleming, N. Mandall, and A. T. Dibiase, "A comparison of two different techniques for early correction of Class III malocclusion," Angle Orthodontist, vol. 82, no. 1, pp. 96-101, 2012.

[41] T. D. Creekmore and M. K. Eklund, "The possibility of skeletal anchorage," Journal of Clinical Orthodontics, vol. 17, no. 4, pp. 266-269, 1983.

[42] A. Enacar, B. Giray, M. Pehlivanoglu, and H. Iplikcioglu, "Facemask therapy with rigid anchorage in a patient with maxillary hypoplasia and severe oligodontia," American Journal of Orthodontics and Dentofacial Orthopedics, vol. 123, no. 5, pp. 571-577, 2003.

[43] M. S. Block and D. R. Hoffman, "A new device for absolute anchorage for orthodontics," American Journal of Orthodontics and Dentofacial Orthopedics, vol. 107, no. 3, pp. 251-258, 1995.

[44] H. Hong, P. Ngan, H. G. Li, L. G. Qi, and S. H. Y. Wei, "Use of onplants as stable anchorage for facemask treatment: a case report," The Angle Orthodontist, vol. 75, no. 3, pp. 453-460, 2005.

[45] W. M. Smalley, P. A. Shapiro, T. H. Hohl, V. G. Kokich, and P.-I. Brånemark, "Osseointegrated titanium implants for maxillofacial protraction in monkeys," American Journal of Orthodontics and Dentofacial Orthopedics, vol. 94, no. 4, pp. 285-295, 1988.

[46] S. L. Singer, P. J. Henry, and I. Rosenberg, "Osseointegrated implants as an adjunct to facemask therapy: a case report," The Angle Orthodontist, vol. 70, no. 3, pp. 253-262, 2000.

[47] B. Sheller and L. Omnell, "Therapeutic ankylosis of primary teeth," Journal of Clinical Orthodontics, vol. 25, no. 8, pp. 499$502,1991$.

[48] M. L. Omnell and B. Sheller, "Maxillary protraction to intentionally ankylosed deciduous canines in a patient with cleft palate," American Journal of Orthodontics and Dentofacial Orthopedics, vol. 106, no. 2, pp. 201-205, 1994.

[49] V. G. Kokich, P. A. Shapiro, R. Oswald, L. Koskinen-Moffett, and S. K. Clarren, "Ankylosed teeth as abutments for maxillary protraction: a case report," American Journal of Orthodontics, vol. 88 , no. 4 , pp. 303-307, 1985.

[50] S. Nevzatoğlu and N. Küçükkeleş, "Long-term results of surgically assisted maxillary protraction vs regular facemask," The Angle Orthodontist, vol. 84, no. 6, pp. 1002-1009, 2014.

[51] C. Liu, M. Hou, L. Liang et al., "Sutural distraction osteogenesis (SDO) versus osteotomy distraction osteogenesis (ODO) for midfacial advancement: a new technique and primary clinical report," Journal of Craniofacial Surgery, vol. 16, no. 4, pp. 537548, 2005.

[52] A. Rachmiel, D. Aizenbud, L. Ardekian, M. Peled, and D. Laufer, "Surgically-assisted orthopedic protraction of the maxilla in cleft lip and palate patients," International Journal of Oral and Maxillofacial Surgery, vol. 28, no. 1, pp. 9-14, 1999.

[53] M. L. Samchukov, Craniofacial Distraction Osteogenesis, Mosby, 2001.

[54] S. Nevzatoğlu and N. Küçükkeleş, "Long-term results of surgically-assisted maxillary protraction," Australian Orthodontic Journal, vol. 30, no. 1, pp. 19-31, 2014.

[55] H. Long, F. Jian, and W. Lai, "Weak evidence supports the short-term benefits of orthopaedic treatment for Class III malocclusion in children," Evidence-Based Dentistry, vol. 15, no. 1, pp. 21-22, 2014. 
[56] H. N. Yilmaz, H. Garip, T. Satilmis, and N. Kucukkeles, "Corticotomy-assisted maxillary protraction with skeletal anchorage and Class III elastics," Angle Orthodontist, vol. 85, no. 1, pp. 48-57, 2015.

[57] K. E. Macdonald, A. J. Kapust, and P. K. Turley, "Cephalometric changes after the correction of class III malocclusion with maxillary expansion/facemask therapy," American Journal of Orthodontics and Dentofacial Orthopedics, vol. 116, no. 1, pp. 1324, 1999.

[58] C. T. Hino, L. H. S. Cevidanes, T. T. Nguyen, H. J. De Clerck, L. Franchi, and J. A. McNamara Jr., "Three-dimensional analysis of maxillary changes associated with facemask and rapid maxillary expansion compared with bone anchored maxillary protraction," American Journal of Orthodontics and Dentofacial Orthopedics, vol. 144, no. 5, pp. 705-714, 2013.

[59] A. Jäger, B. Braumann, C. Kim, and S. Wahner, "Skeletal and dental effects of maxillary protraction in patients with angle class III malocclusion: a meta-analysis," Journal of Orofacial Orthopedics, vol. 62, no. 4, pp. 275-284, 2001.

[60] M. Celikoglu and H. Oktay, "Effects of maxillary protraction for early correction of class III malocclusion," The European Journal of Orthodontics, vol. 36, no. 1, pp. 86-92, 2014.

[61] G. Cordasco, G. Matarese, L. Rustico et al., "Efficacy of orthopedic treatment with protraction facemask on skeletal Class III malocclusion: a systematic review and meta-analysis," Orthodontics \& Craniofacial Research, vol. 17, no. 3, pp. 133-143, 2014.

[62] E.-W. Liou and W.-C. Tsai, "A new protocol for maxillary protraction in cleft patients: repetitive weekly protocol of alternate rapid maxillary expansions and constrictions," Informationen aus Orthodontie \& Kieferorthopädie, vol. 39, no. 4, pp. 267-274, 2007.

[63] Y.-C. Wang, P. M. S. Chang, and E. J.-W. Liou, "Opening of circumaxillary sutures by alternate rapid maxillary expansions and constrictions," The Angle Orthodontist, vol. 79, no. 2, pp. 230-234, 2009.

[64] O. G. da Silva Filho, A. C. Magro, and L. Capelozza Filho, "Early treatment of the class III malocclusion with rapid maxillary expansion and maxillary protraction," American Journal of Orthodontics and Dentofacial Orthopedics, vol. 113, no. 2, pp. 196-203, 1998.

[65] M. Nienkemper, B. Wilmes, A. Pauls, and D. Drescher, "Maxillary protraction using a hybrid hyrax-facemask combination," Progress in Orthodontics, vol. 14, no. 1, pp. 1-8, 2013.

[66] M. D. Williams, D. M. Sarver, P. L. Sadowsky, and E. Bradley, "Combined rapid maxillary expansion and protraction facemask in the treatment of Class III malocclusions in growing children: a prospective long-term study." Seminars in Orthodontics, vol. 3, no. 4, pp. 265-274, 1997.

[67] G. C. Heymann, L. Cevidanes, M. Cornelis, H. J. De Clerck, and J. F. C. Tulloch, "Three-dimensional analysis of maxillary protraction with intermaxillary elastics to miniplates," American Journal of Orthodontics and Dentofacial Orthopedics, vol. 137, no. 2, pp. 274-284, 2010.

[68] K. Takada, S. Petdachai, and M. Sakuda, "Changes in dentofacial morphology in skeletal Class III children treated by a modified maxillary protraction headgear and a chin cup: A longitudinal cephalometric appraisal," European Journal of Orthodontics, vol. 15, no. 3, pp. 211-221, 1993.

[69] J. Lim and Y. Park, "A study on profile change of skeletal Class III malocclusion patients after wearing protraction headgear," Korean Journal of Orthodontics, vol. 25, pp. 375-401, 1995.
[70] F. Janssens, G. Swennen, T. Dujardin, R. Glineur, and C. Malevez, "Use of an onplant as orthodontic anchorage," American Journal of Orthodontics and Dentofacial Orthopedics, vol. 122, no. 5, pp. 566-570, 2002.

[71] W. K. Tsui, H. D. P. Chua, and L. K. Cheung, "Bone anchor systems for orthodontic application: a systematic review," International Journal of Oral and Maxillofacial Surgery, vol. 41, no. 11, pp. 1427-1438, 2012.

[72] T. Baccetti, H. J. De Clerck, L. H. Cevidanes, and L. Franchi, "Morphometric analysis of treatment effects of bone-anchored maxillary protraction in growing Class III patients," The European Journal of Orthodontics, vol. 33, no. 2, pp. 121-125, 2011.

[73] B. H. Kircelli and Z. Ö. Pektas, "Midfacial protraction with skeletally anchored face mask therapy: a novel approach and preliminary results," American Journal of Orthodontics and Dentofacial Orthopedics, vol. 133, no. 3, pp. 440-449, 2008.

[74] H. J. De Clerck, M. A. Cornelis, L. H. Cevidanes, G. C. Heymann, and C. J. F. Tulloch, "Orthopedic traction of the maxilla with miniplates: a new perspective for treatment of midface deficiency," Journal of Oral and Maxillofacial Surgery, vol. 67, no. 10, pp. 2123-2129, 2009.

[75] S.-H. Baek, K.-W. Kim, and J.-Y. Choi, "New treatment modality for maxillary hypoplasia in cleft patients: protraction facemask with miniplate anchorage," The Angle Orthodontist, vol. 80, no. 4, pp. 783-791, 2010.

[76] M. Morales-Fernández, A. Iglesias-Linares, R. M. YañezVico, A. Mendoza-Mendoza, and E. Solano-Reina, "Bone- and dentoalveolar-anchored dentofacial orthopedics for Class III malocclusion: new approaches, similar objectives? A systematic review," Angle Orthodontist, vol. 83, no. 3, pp. 540-552, 2013.

[77] T. Nguyen, L. Cevidanes, M. A. Cornelis, G. Heymann, L. K. de Paula, and H. de Clerck, "Three-dimensional assessment of maxillary changes associated with bone anchored maxillary protraction," American Journal of Orthodontics and Dentofacial Orthopedics, vol. 140, no. 6, pp. 790-798, 2011.

[78] H. De Clerck, L. Cevidanes, and T. Baccetti, "Dentofacial effects of bone-anchored maxillary protraction: a controlled study of consecutively treated class III patients," American Journal of Orthodontics and Dentofacial Orthopedics, vol. 138, no. 5, pp. 577-581, 2010.

[79] Ç. Şar, A. Arman-Özçırpıcı, S. Uçkan, and A. C. Yazıcı, "Comparative evaluation of maxillary protraction with or without skeletal anchorage," American Journal of Orthodontics and Dentofacial Orthopedics, vol. 139, no. 5, pp. 636-649, 2011.

[80] P. Cozza, T. Baccetti, M. Mucedero, C. Pavoni, and L. Franchi, "Treatment and posttreatment effects of a facial mask combined with a bite-block appliance in Class III malocclusion," American Journal of Orthodontics and Dentofacial Orthopedics, vol. 138, no. 3, pp. 300-310, 2010.

[81] I. Yoshida, T. Shoji, and I. Mizoguchi, "Effects of treatment with a combined maxillary protraction and chincap appliance in skeletal class III patients with different vertical skeletal morphologies," The European Journal of Orthodontics, vol. 29, no. 2, pp. 126-133, 2007.

[82] X. Yan, W. He, T. Lin et al., "Three-dimensional finite element analysis of the craniomaxillary complex during maxillary protraction with bone anchorage vs conventional dental anchorage," American Journal of Orthodontics and Dentofacial Orthopedics, vol. 143, no. 2, pp. 197-205, 2013.

[83] T. Baccetti, L. Franchi, M. Mucedero, and P. Cozza, "Treatment and post-treatment effects of facemask therapy on the sagittal 
pharyngeal dimensions in Class III subjects," The European Journal of Orthodontics, vol. 32, no. 3, pp. 346-350, 2010.

[84] M. A. Ghiz, P. Ngan, and E. Gunel, "Cephalometric variables to predict future success of early orthopedic class III treatment," American Journal of Orthodontics and Dentofacial Orthopedics, vol. 127, no. 3, pp. 301-306, 2005.

[85] K. Hirata, S. Haraguchi, W. Tome, and T. Yamashiro, "Twophase treatment in a patient with a skeletal Class III malocclusion: early orthopedic treatment and subsequent camouflage treatment with temporary anchorage devices," Orthodontic Waves, vol. 73, no. 3, pp. 102-109, 2014.

[86] G. T. McIntyre and P. A. Mossey, "Size and shape measurement in contemporary cephalometrics," European Journal of Orthodontics, vol. 25, no. 3, pp. 231-242, 2003.

[87] T. Baccetti, L. Franchi, and J. A. McNamara Jr., "Thin-plate spline analysis of treatment effects of rapid maxillary expansion and face mask therapy in early class III malocclusions," European Journal of Orthodontics, vol. 21, no. 3, pp. 275-281, 1999.

[88] C. J. Lux, J. Rübel, J. Starke, C. Conradt, A. Stellzig, and G. Komposch, "Effects of early activator treatment in patients with class II malocclusion evaluated by thin-plate spline analysis," Angle Orthodontist, vol. 71, no. 2, pp. 120-126, 2001.

[89] N. Küçükkeleş, Ş. Nevzatoǧlu, and T. Koldaş, "Rapid maxillary expansion compared to surgery for assistance in maxillary face mask protraction," Angle Orthodontist, vol. 81, no. 1, pp. 44-51, 2011.

[90] J. C. Palma, N. Tejedor-Sanz, M. D. Oteo, and J. A. Alarcón, "Long-term stability of rapid maxillary expansion combined with chincup protraction followed by fixed appliances," Angle Orthodontist, vol. 85, no. 2, pp. 270-277, 2015.

[91] P. V. Westwood, J. A. McNamara Jr., T. Baccetti, L. Franchi, and D. M. Sarver, "Long-term effects of Class III treatment with rapid maxillary expansion and facemask therapy followed by fixed appliances," American Journal of Orthodontics and Dentofacial Orthopedics, vol. 123, no. 3, pp. 306-320, 2003.

[92] U. Hägg, A. Tse, M. Bendeus, and A. B. M. Rabie, "Long-term follow-up of early treatment with reverse headgear," European Journal of Orthodontics, vol. 25, no. 1, pp. 95-102, 2003.

[93] K. Tahmina, E. Tanaka, and K. Tanne, "Craniofacial morphology in orthodontically treated patients of Class III malocclusion with stable and unstable treatment outcomes," American Journal of Orthodontics and Dentofacial Orthopedics, vol. 117, no. 6, pp. 681-690, 2000.

[94] A. Zentner, G. M. Doll, and S. M. Peylo, "Morphological parameters as predictors of successful correction of Class III malocclusion," The European Journal of Orthodontics, vol. 23, no. 4, pp. 383-392, 2001. 


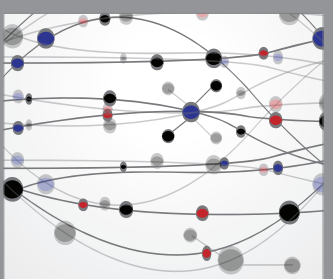

The Scientific World Journal
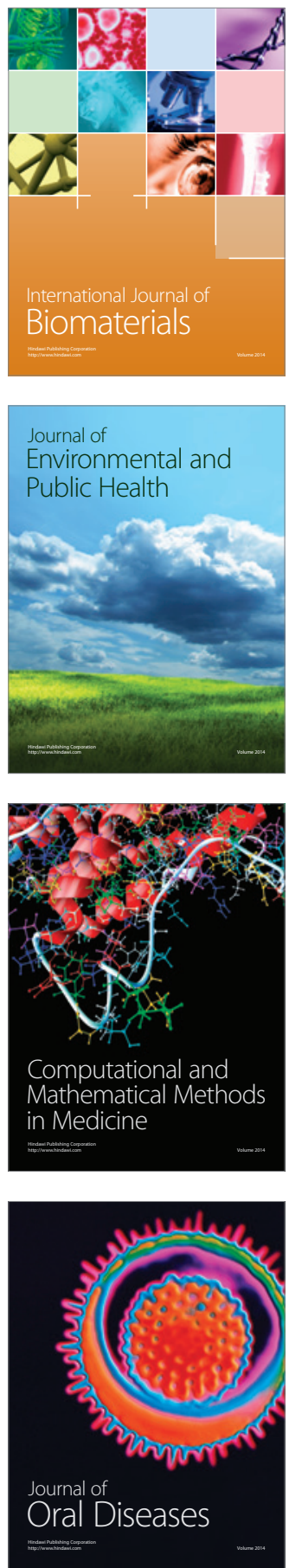
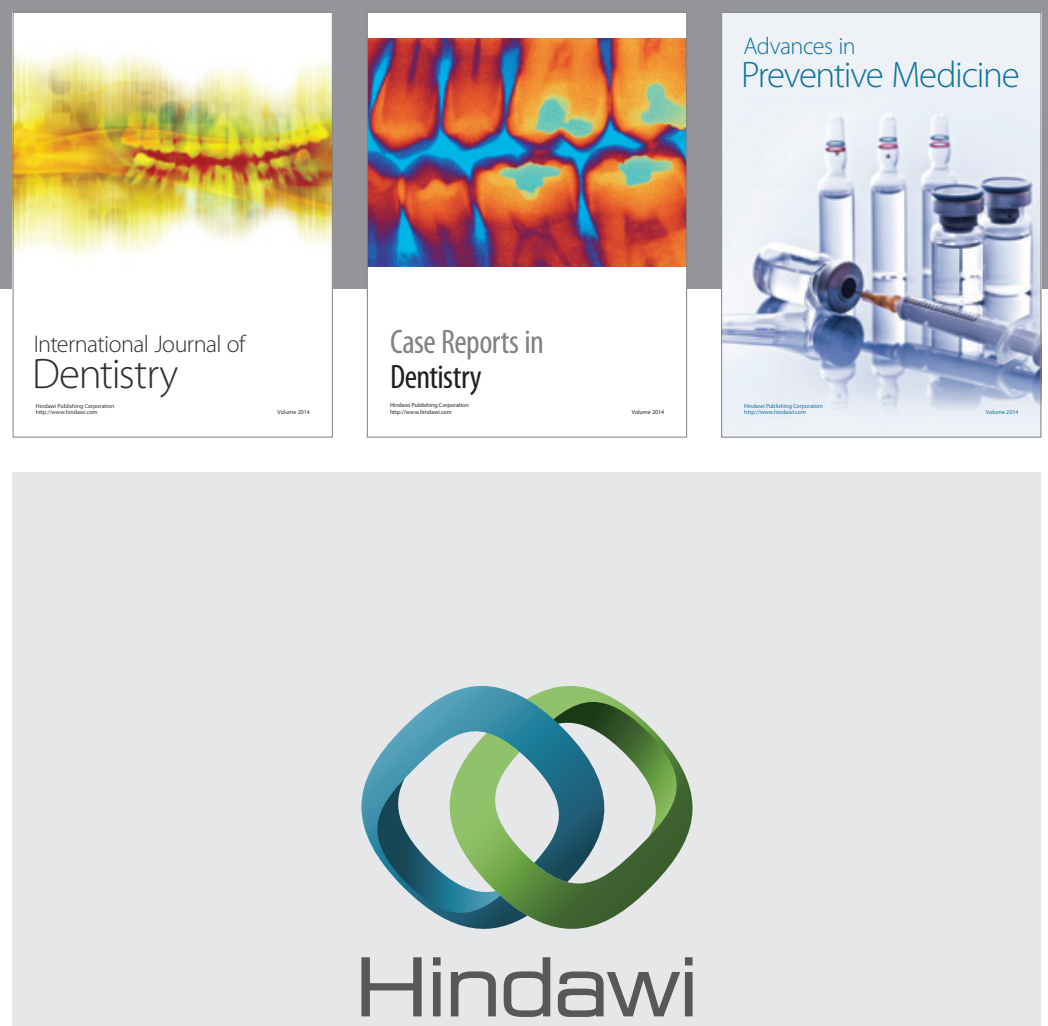

Submit your manuscripts at

http://www.hindawi.com
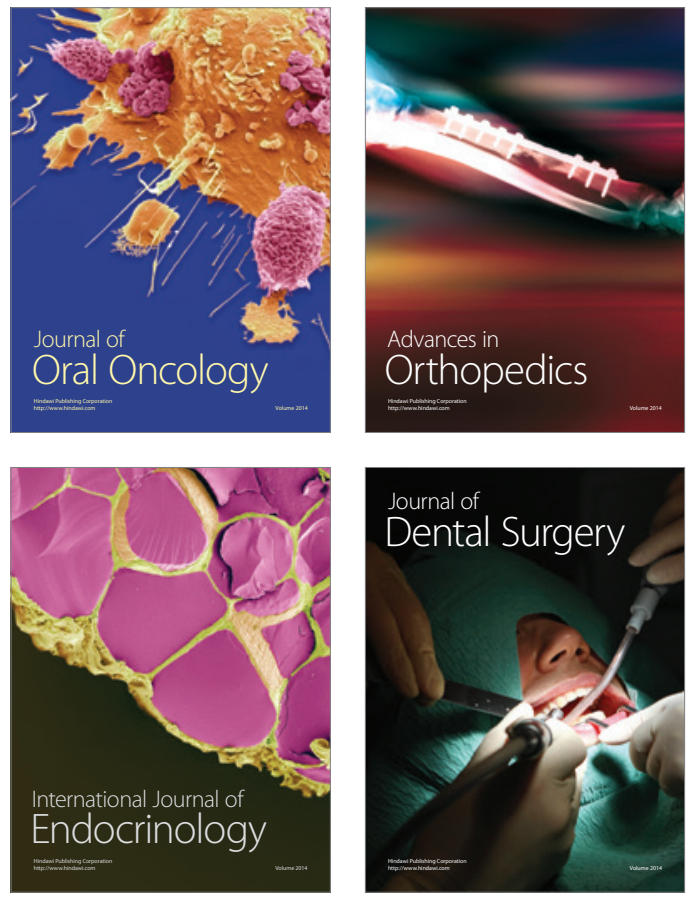
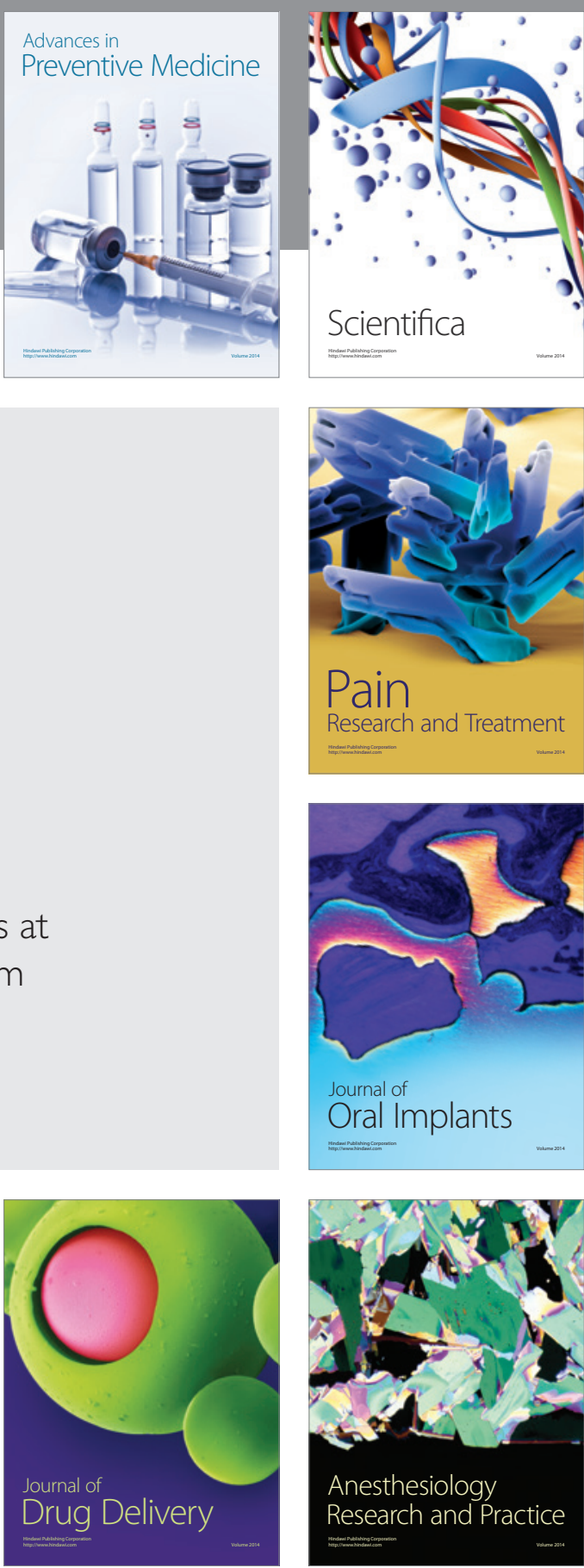

Scientifica
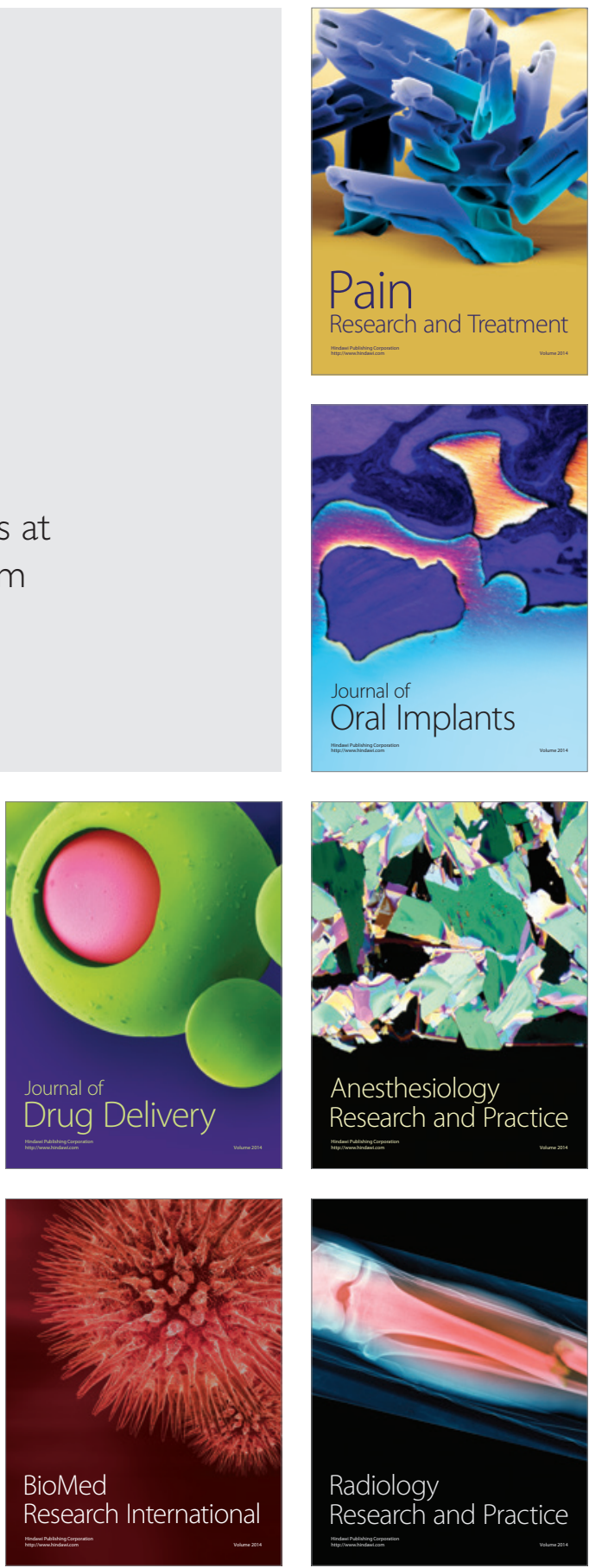